\title{
Integrated Multi-Dithering Controller for Adaptive Optics
}

\author{
Dimitrios N. Loizos*, Ling Liu ${ }^{\dagger}$, Paul P. Sotiriadis*, Gert Cauwenberghs ${ }^{\ddagger}$ and Mikhail A. Vorontsov ${ }^{\dagger \S}$ \\ *Department of Electrical and Computer Engineering, The Johns Hopkins University, Baltimore, MD 21218 \\ ${ }^{\dagger}$ Intelligent Optics Laboratory, Institute for Systems Research, University of Maryland, College Park, MD 20742 \\ ${ }^{\ddagger}$ Division of Biological Sciences, University of California, San Diego, CA 92093 \\ ${ }^{\S}$ Computational and Information Sciences Directorate, U.S. Army Research Laboratory, Adelphi, MD 20783
}

\begin{abstract}
Effective compensation of phase noise in laser communication calls for fast, real-time, adaptive wavefront control. We present an analog, continuous-time, high-speed VLSI (Very Large Scale Integration) controller implementing multi-dithering perturbative gradient descent optimization of a direct measure of optical performance. The system applies parallel sinusoidal perturbations to the wavefront over a range of frequencies, and performs parallel synchronous detection of the metric signal to derive the gradient components over each frequency band. The system operates over a wide range of frequencies, supporting applications of model-free adaptive optics extending from compensation of slow atmospheric turbulence to compensation of fast random phase fluctuations in the actuators and laser amplifiers. The system has been tested as a phase controller for a multiple laser beam wavefront propagating through a highly turbulent medium. The results indicate a compensation bandwidth exceeding $300 \mathrm{kHz}$ matching the turbulence bandwidth.
\end{abstract}

\section{INTRODUCTION}

Optical communications between a single source, $N$-transducer transmitting array and a single receiver (Fig. 1) can be significantly impaired due to phase noise introduced in the system. Typical examples of sources that alter the phase of each channel and lead to non-coherent detection of the combined signal at the receiver are atmospheric turbulence [1]-[4], phase noise of the laser source itself, as well as phase noise introduced by the fiber amplifiers [5]. These sources can be characterized by a highly non-linear and time-varying behavior, featuring bandwidths that can exceed a few MHz. The need for high-speed adaptive controllers for phase correction is therefore apparent, and significant efforts in the optical engineering community have been directed towards increasing bandwidth and precision of adaptive optics control systems, e.g. [6], [7].

Analog Very Large Scale Integrated (VLSI) circuits have received a lot of attention as a candidate means of implementing adaptive controllers due to their advantages compared to software and discrete-component solutions: low-power consumption, compact size, faster computation and control of multiple channels in a smaller area. Effective compensation of scintillation caused by atmospheric turbulence has been demonstrated [7], [8] by using analog VLSI chips implementing the stochastic parallel gradient descent (SPGD) optimization technique for bandwidths up to several hundreds of Hz. A challenging task has been to opt the adaptation speeds of such controllers, by possibly implementing different algorithms in VLSI, in order to be able to compensate higher bandwidth sources of phase distortion.

A proposed alternative to the SPGD algorithm has been sinusoidal multi-dithering, so far demonstrated using only discretecomponent hardware [9], [10]. The distinctive difference between the two methods lies in the choice of the dithers superimposed to the controlling signals of the phases; for SPGD, a small-amplitude binary random sequence is applied to each control variable, whereas in sinusoidal multi-dithering the dithers are sinusoidal signals of distinct frequencies, different for each variable. Due to the very nature of the two algorithms, there are advantages in applying the one over the other, according to the specific application. Stochastic algorithms require less hardware since they are computationally more efficient and are, therefore, more appealing when easy integration of multiple channels is needed. On the other hand, sinusoidal multi-dithering achieves faster adaptation and can compensate for higher bandwidth distortion.

A multi-dithering parallel architecture for adaptive phase correction in optical systems, its implementation in analog VLSI circuits, and its experimental demonstration in an optical power combining application, are presented in this paper. The architecture consists of 8 channels for control of up to 8 phase variables and can be tiled in order to incorporate more channels. Moreover, the architecture features additional circuitry in order to address issues pertinent to the control of phase and fabrication non-idealities of the phase shifters. More specifically:

- The dynamic range of the control variables (phases) is limited within programmable limits that correspond to the operating region of the phase shifters. Saturation is avoided by continuously monitoring the values of the control variables and performing voltage jumps equivalent to $2 \pi$ phase shifts, whenever the controlled variables try to exceed the limits. The observed metric (received power, SNR, etc.) is not affected as long as the jumps are instantaneous, due to its modulo- $2 \pi$ relation to the controlled phases. 


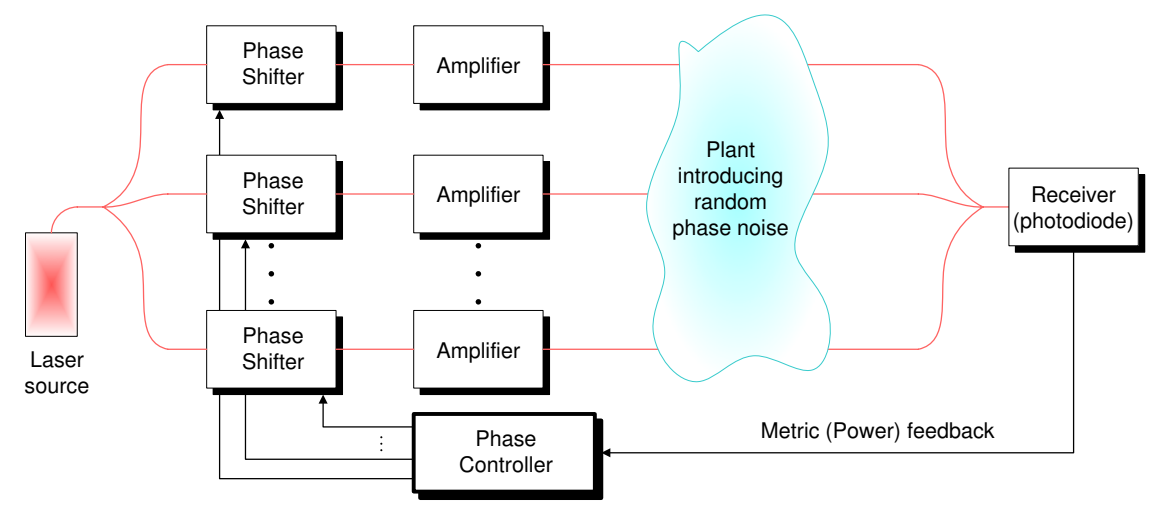

Fig. 1. General considered setup. A controller is needed to adjust the signals that pre-distort the phase of the beams at each channel, in order to compensate distortion later introduced by the unknown and time-varying plant.

- The common mode of all controlled variables is adjusted and also constrained within programmable limits. In this way, common mode drift across the channels is avoided.

Operation of the controller has been tested in an all-fiber optical setup and the performance has been measured by observing the power of the combined optical signals at the receiver. The relation between the controlled phases and the observed power metric is provided in Section II-A. Suppression of in-loop phase distortion and equivalent power maximization is demonstrated for bandwidths up to $300 \mathrm{kHz}$.

\section{Metric Optimization}

The objective of multi-dithering gradient descent control is to adaptively minimize a supplied metric of optical performance, by measuring the gradient of the metric with respect to the control variables through parallel dithering [9]. Before presenting the implemented architecture, we define and review the metric used here for optical beam power combining, and show how the well-known multi-dithering approach to gradient estimation extends to $L_{1}$-norm minimization of the metric through rectification of the gradient estimate.

As a general framework, the setup of Fig. 1 is considered. A single laser source is split uniformly into $N$ channels, so as to take advantage of power redistribution and maximization at a single spot during coherent combination at the receiver. The beam at each channel is amplified and then appropriately directed at a center spot in the receiver. The beam positioning mechanism is beyond the scope of this paper and will not be addressed. An unknown and time-varying plant introduces a random, time-varying phase shift to each beam causing light combination at random coherence levels in the receiver. In order to ensure power maximization, a feedback mechanism is employed that uses the information of received power and pre-distorts the phase at each channel of the transmitter, so as to compensate, in continuous-time, phase distortions later introduced by the plant.

\section{A. Power Calculation}

Let us assume a single optical source driving $N$ transmitters aligned towards the same receiver [11], through an unknown and turbulent propagation medium ("plant") as shown in Fig. 1, and denote the wavefunction of light at the receiver, from each transmitter, as

$$
a_{i}\left(U_{i}, t\right)=A_{i} e^{j U_{i}} e^{j 2 \pi f_{c} t}, i=1, \ldots, n
$$

where $A_{i}$ is the amplitude, $U_{i}$ is the phase at which light arrives to the receiver from source $i$, and $f_{c}$ is the frequency of light emitted by the source.

The resulting optical wave $a(\mathbf{U}, t)$ at the receiver is the superposition of all wavefunctions $a_{i}\left(U_{i}, t\right)$

$$
a(\mathbf{U}, t)=\sum_{i=1}^{n} A_{i} e^{j U_{i}} e^{j 2 \pi f_{c} t}
$$

and the total average power at the receiver will be proportional to

$$
P(\mathbf{U}) \propto \Re\left\{\frac{a(\mathbf{U}, t) \cdot a^{*}(\mathbf{U}, t)}{2}\right\}=\sum_{i, j} A_{i} A_{j} \cos \left(U_{i}-U_{j}\right) .
$$

Power is maximized when all phase differences $U_{i}-U_{j}$ are multiples of $2 \pi$, i.e., when the optical waves from all $N$ transmitters are coherently combined at the receiver. 


\section{B. Multi-Dithering}

Sinusoidal multi-dithering in optics was first introduced by O'Meara [9] as a method for coherent adaptive optics. Here, the basic principles will be reviewed and the way multi-dithering can be combined with the gradient descent (ascent) technique in order to achieve optimization will be clarified. Moreover the advantages of using the sinusoidal multi-dithering technique over other optimization techniques will be presented.

1) The Gradient Descent Algorithm: The gradient descent algorithm, known in its continuous-time form also as gradient descent flow, drives a scalar metric $J$ controlled by variables $\mathbf{u}=\left(u_{1}, u_{2}, \ldots, u_{n}\right)^{\mathrm{T}}$ to a (local) minimum, if such exists, by updating $\mathbf{u}$ according to the following rule

$$
\frac{d \mathbf{u}}{d t}=-G \nabla J(\mathbf{u})
$$

where $G>0$ is a constant that adjusts the convergence speed. A variant form of (1), easier to implement in hardware, requires only the information of the sign of the partial derivative components of the gradient $\nabla J$

$$
\frac{d u_{i}}{d t}=-G \operatorname{sgn}\left(\frac{\partial J}{\partial u_{i}}\right), i=1, \ldots, n \text {. }
$$

Proof of convergence for the above rule is achieved by expanding the time derivative of $J$ and using the chain rule

$$
\frac{d}{d t} J(\mathbf{u}(t))=(\nabla J(\mathbf{u}))^{\mathrm{T}} \frac{d \mathbf{u}}{d t}=-G|\nabla J|_{1},
$$

where $|\nabla J|_{1}=\sum_{i=1}^{n}\left|\frac{\partial J}{\partial u_{i}}\right|$ denotes the L-1 norm of $\nabla J$. According to (3), since $|\nabla J|_{1}$ will be always non-negative, $J$ will continuously move towards a (local) minimum.

2) Sinusoidal Multi-Dithering: Multi-dithering relies on perturbing in parallel all variables $u_{i}$ that control a metric $J(\mathbf{u})$ in order to estimate the gradient of $J$. It is a model-free [12] adaptive algorithm and therefore requires minimum knowledge about the system on which it is applied, making it ideal for applications where the under optimization plant is time-varying and described by highly nonlinear state equations. In sinusoidal multi-dithering, the dithers that perturb $u_{i}$ are sinusoidal signals of different frequencies $\omega_{i}$ for each variable and of small amplitude $\alpha$

$$
\tilde{u}_{i}=u_{i}+\delta u_{i}=u_{i}+\alpha \cos \left(\omega_{i} t\right), i=1, \ldots, n
$$

Parallel coherent detection between the perturbed performance metric $J$ and the dithers at each channel leads to an estimate of the partial derivatives $\frac{\partial J}{\partial u_{i}}$, and therefore the gradient

$$
\overline{J(\tilde{\mathbf{u}}) \cos \left(\omega_{i} t\right)}=\frac{\alpha}{2} \frac{\partial J}{\partial u_{i}}, i=1, \ldots, n
$$

where the overline denotes low-pass filtering. More details on how coherent detection can lead to the above expression can be found in [9], [13].

\section{SyStem ARChiteCTURE}

\section{A. General description}

The block diagram of a single channel for the phase controller is shown in Fig. 2. A 3-phase oscillator generates the dithers that perturb the control variables $u_{i}$ at distinct frequencies $\omega_{i}$. The amplitude of the dithers can be tuned using the capacitor array preceding the output of the channel. The perturbed variable $\tilde{u}_{i}=u_{i}+\alpha \cos \left(\omega_{i} t\right)$ is applied as a control signal to the $i$-th phase shifter in Fig. 1. The power metric is evaluated and then provided back to the controller. At the front-end of the controller the dither is used for synchronous detection - multiplication and low-pass filtering - of the received metric. The output of the low-pass filter, as shown in Section II-B.2, is proportional to the partial derivative $\frac{\partial J(\mathbf{u})}{\partial u_{i}}$ element of the gradient $\nabla J$. The comparator quantizes the gradient information and an XOR gate is used to select between minimization or maximization of the under optimization metric. Finally, a charge pump updates $u_{i}$, which is stored across capacitor $C$. Further details on the circuit implementation of the blocks can be found in [14].

\section{B. Multiple $2 \pi$ Phase Control}

Phase distortions in the unknown plant can dictate continuous increase or decrease of the predistorting phase shift added by the controller, over multiple $2 \pi$ cycles. Considering candidate phase shifters, spatial light modulators (SLMs), such as Pockels Readout Modulators (PROM) [15], microchannel SLMs [16], light valves that use $\mathrm{KD}_{2} \mathrm{PO}_{4}$ crystals and Fabry-Perot modulators [17] offer very high resolution [18], however require control voltages in the $\mathrm{kV}$ range and have slow responses (hundreds of ms) [16]. On the other hand, Lithium Niobate $\left(\mathrm{LiNbO}_{3}\right)$ phase modulators have bandwidths that exceed $40 \mathrm{GHz}$ [19], nonetheless suffer from drift of the phase corresponding to a specific control voltage and from lower resolution compared 


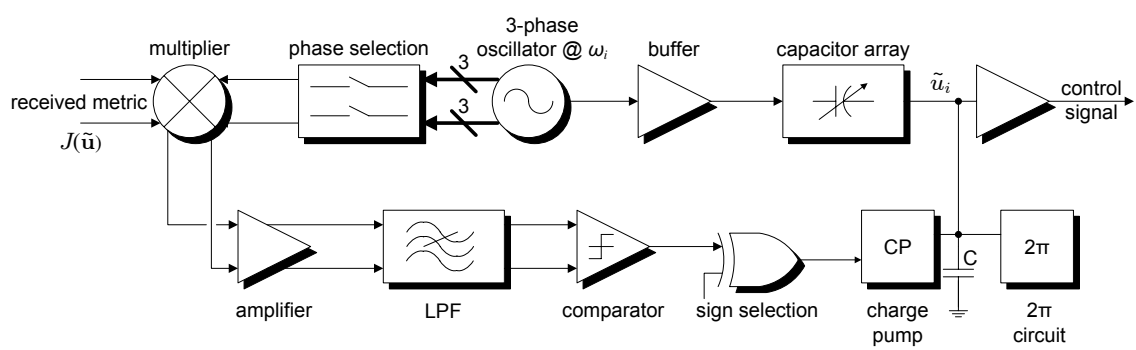

Fig. 2. Block diagram architecture of a single control channel. The metric is shared by all channels in the system.

to SLMs. Since drift can be compensated by the controller and resolution is less of a concern than bandwidth for our specific application, $\mathrm{LiNbO}_{3}$ phase modulators were chosen for the implementation.

Irrespective of their type, all phase modulators have a limited range of achievable phase shifts, limited by saturation values on the upper and lower end; typically, the operating range spans a few $2 \pi$ regions. A technique that has been proposed for adaptive optics applications in order to compensate for saturation is the use of the Interference Phase Loop (IPL) [18], [20], [21]. In the IPL, the feedback signal used to control the phase modulator and achieve phase compensation is the intensity of the interference between the received signal and a reference signal. Since the interference intensity is proportional to the sine of the phase difference between reference and received signal, the control signal of the phase modulator will be always bounded, and the bounds can be chosen to be absolutely lower than the saturation values.

The IPL method has been so far demonstrated for compensation of only a single phase. In this work we have been primarily concerned about techniques that can be applied along with the multi-dithering algorithm for phase compensation in a multiple channel architecture. The approach that has been followed is illustrated in Fig. 3. The control signal $u_{i}$ of each phase modulator, which is the voltage across capacitor $C$ (Fig. 2) of the charge pump at every channel, is monitored by a circuit, further referred to as the " $2 \pi$ circuit". The " $2 \pi$ circuit" compares $u_{i}$ to programmable upper and lower bounds, $v_{+2 \pi}$ and $v_{-2 \pi}$ respectively, and whenever $u_{i}$ exceeds these limits, its value is reset to $v_{0}$. The voltage differences $v_{+2 \pi}-v_{0}$ and $v_{0}-v_{-2 \pi}$ should be set so as to correspond to $2 \pi$ phase shifts in the phase modulator. The reset operation should be ideally instantaneous. The idea behind this approach lies on the fact that the received power is proportional to $\sum_{i \neq j} \cos \left(u_{i}-u_{j}\right)$ and therefore instantaneous $2 \pi$ jumps in either $u_{i}$ or $u_{j}$ will not affect the power. It should be noted that the voltage differences $v_{+2 \pi}-v_{0}$ and $v_{0}-v_{-2 \pi}$ can be set to correspond to any multiple of $2 \pi$ as long as $v_{+2 \pi}$ and $v_{-2 \pi}$ fall within the linear operating region of the phase modulator.

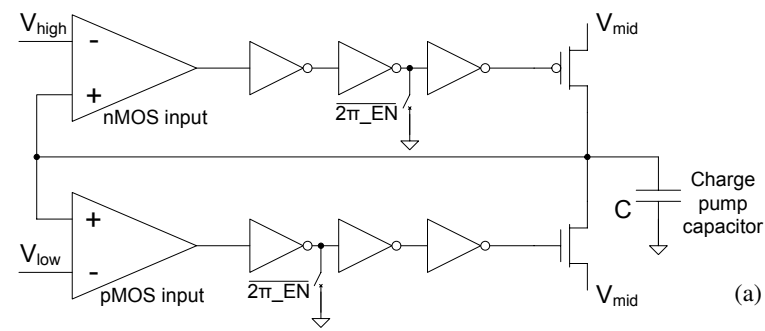

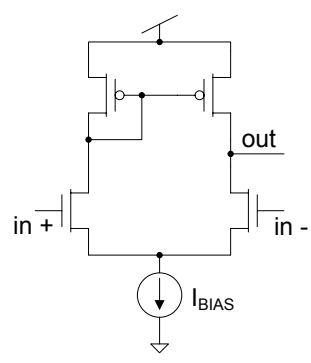

(b)

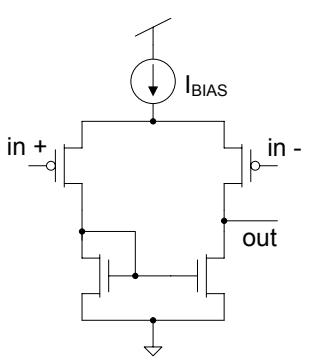

(c)

Fig. 3. (a) Block diagram architecture of the $2 \pi$ circuit (b) Comparator architecture with nMOS input (c) Comparator architecture with pMOS input.

In practice, the reset time is not instantaneous and depends both on the value of capacitor $C$ as well as the differences $v_{+2 \pi}-v_{0}$ and $v_{0}-v_{-2 \pi}$. Inverters functioning as delay elements have been added to the design in order to provide sufficient time for the control value to be reset before the nMOS or pMOS current source gets disabled. The operation of the circuit can 
be suspended by setting the signal $2 \pi$ EN low.

A simple 5-transistor design has been implemented for both comparators, as shown in Figs. 3(b) and (c). Input with nMOS (pMOS) transistors has been chosen for comparison with the upper (lower) threshold $v_{+2 \pi}\left(v_{-2 \pi}\right)$ so that these bounds can take values up to the positive (negative) rail.

In order to further clarify the operation of the " $2 \pi$ circuit", let us assume the case of Fig. 4, where sinusoidal phase noise of amplitude $3 \pi$ has been considered to be introduced in one channel and needs to be compensated by the phase controller. The figure depicts the resulting output phase from the modulator assuming that $v_{0}$ corresponds to 0 rads, $v_{+2 \pi}$ to $2 \pi$ rads and $v_{-2 \pi}$ to $-2 \pi$ rads. The modulo $2 \pi$ phase difference between noise and control is always 0 .

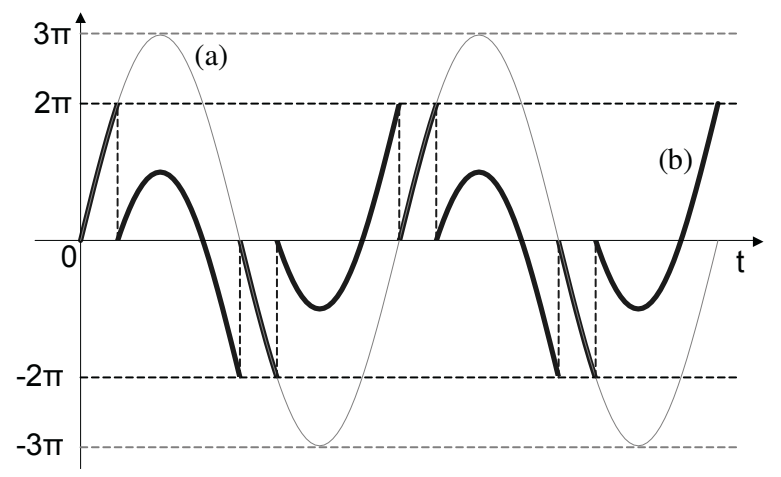

Fig. 4. Example of the ideal response (b) of the controller when trying to track a sinusoidal phase shift (a) of amplitude $3 \pi$. The controller output will be bounded between $-2 \pi$ and $2 \pi$, however, its modulo $2 \pi$ difference from the externally applied phase shift will be always 0 .

\section{Common Mode Adjustment}

Common-mode adjustment is external to the VLSI system, which however provides the average value of all controlled variables, and has been embedded to the testing board that provides an interface between the system and the phase shifters where the control signals are applied. A simplified schematic of the circuit is shown in Fig. 5, where avg is the average of all controlled variables provided from the VLSI system, avg $g_{\text {high }}$ and $\operatorname{avg}_{\text {low }}$ are programmable thresholds and $\mathrm{V}_{\text {source }}$ and $\mathrm{V}_{\text {sink }}$ are the voltage biases for the current sources used for updates in the charge pump.

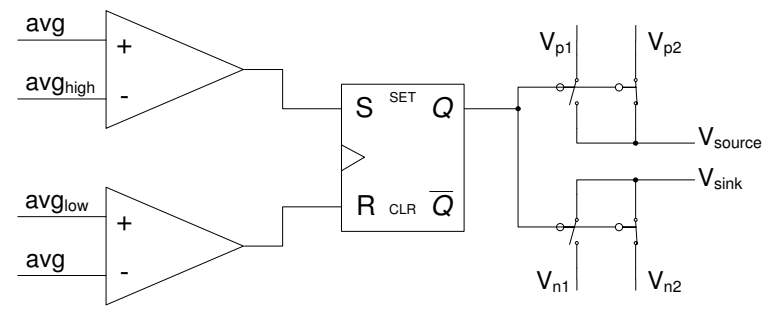

Fig. 5. Circuit for common-mode adjustment of the controlled phases.

According to whether the average value of the phases is above or below the defined thresholds, the state of the S-R flip-flop is changed and either the first or second pair of biasing values is selected. The biases $V_{n i}$ and $V_{p i}$ are set in such a way so that in one state the source current is higher than the sink current and vice versa.

\section{ExPERIMEntal Setup And Measurements}

In order to interface the VLSI system with the optical phase shifters, a test board was fabricated. Special attention was paid so that all inputs and outputs were matched to $50 \Omega$ and thus achieve maximum power transfer for the controlling signals, bearing dither frequencies up to $1 \mathrm{GHz}$. The biasing currents setting the operating point of the system were externally adjusted through a PC. The biases need to be set once and according to the bandwidth of the system, the number of channels, the desired dither frequencies and slew rate of adaptation. Fig. 6 shows a picture of the testing board.

Laboratory experiments in a polarization-maintaining (PM) fiber optic setup (Fig. 7) were performed to validate theory and measure the performance of the controller. Results of free-space experiments using the specific controller can be found in [22]. The source in the fiber setup was a diode laser, emitting light at a wavelength of $1552.93 \mathrm{~nm}$ with a PM fiber-coupled output. Reflections from next stages of the setup back to the source were blocked using an optical isolator. Light was then split into 


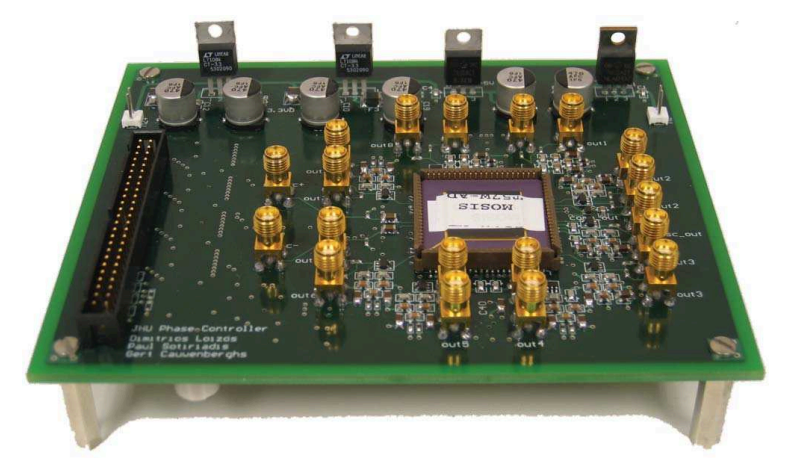

Fig. 6. Test board used as an interface between the VLSI controller and the optical phase shifters. Biasing is externally provided through a PC.

up to 4 channels and phase pre-distorted using a $\mathrm{LiNbO}_{3}$ phase modulator $\left(V_{\pi}=3 \mathrm{~V}\right)$ driven by the multi-dithering controller. Phase pre-distortion was applied in order to compensate for phase distortion later introduced in the optical path. The controlling signals were applied to the phase modulator through broadband, high-speed inverting amplifiers.

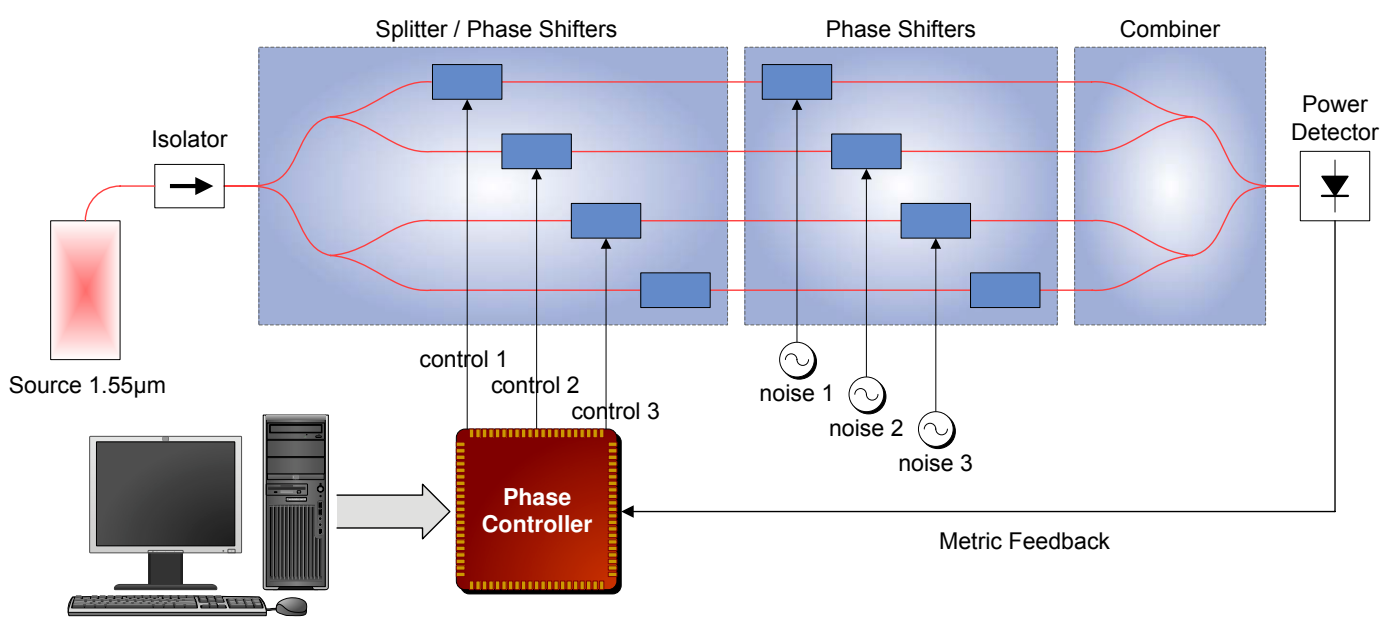

Fig. 7. Experimental setup. An external PC just sets the biasing levels of the different blocks in the chip. Phase distortion is introduced in the path through external noise sources (signal generators). An extra channel with 0 introduced phase shift is used as a reference.

The above setup provides an ideal environment in order to study the effects of phase noise in optical communications, since other issues common to free-space communications and atmospheric turbulence, such as beam spreading and beam wander, are suppressed due to the very nature of fibers. In the setup of Fig. 7, phase noise was emulated by introducing phase shifts to each of the channels, through $\mathrm{LiNbO}_{3}$ phase modulators $\left(V_{\pi}=1.55 \mathrm{~V}\right)$ actuated by external sources.

The loop was closed by combining all channels and measuring the resulting optical power via a PIN photo-diode. The photo-diode's output voltage was used as the metric of the system and fed back to the controller. Further details on the optical components of the system can be found in [23]. The experiments focused in simultaneously introducing time-varying phase distortions to the optical channels and testing the response of the multi-dithering controller in trying to cancel these distortions and optimize power.

Denoting as $u_{i}$ the phase shifts applied to the optical paths from the controller and as $u_{i}^{\prime}$ the phase distortions from the external sources, the total phase shift introduced to each channel is $U_{i}=u_{i}+u_{i}^{\prime}$. According to the results of Section II-A, power is maximized when

$$
\left(U_{i}-U_{j}\right) \bmod 2 \pi=0 \Leftrightarrow\left(U_{i} \bmod 2 \pi\right)-\left(U_{j} \bmod 2 \pi\right)=0, \forall i, j, i \neq j
$$

Applying the variable transformation $x_{i}=\left(U_{i} \bmod 2 \pi\right)$, (4) can be rewritten as

$$
x_{i}-x_{j}=0 \Leftrightarrow x_{i}=x_{j}, \forall i, j, i \neq j
$$

Equation (5) implies that all variables $x_{i}$ will have the same value $c$, which, however, is undetermined. Applying the inverse 

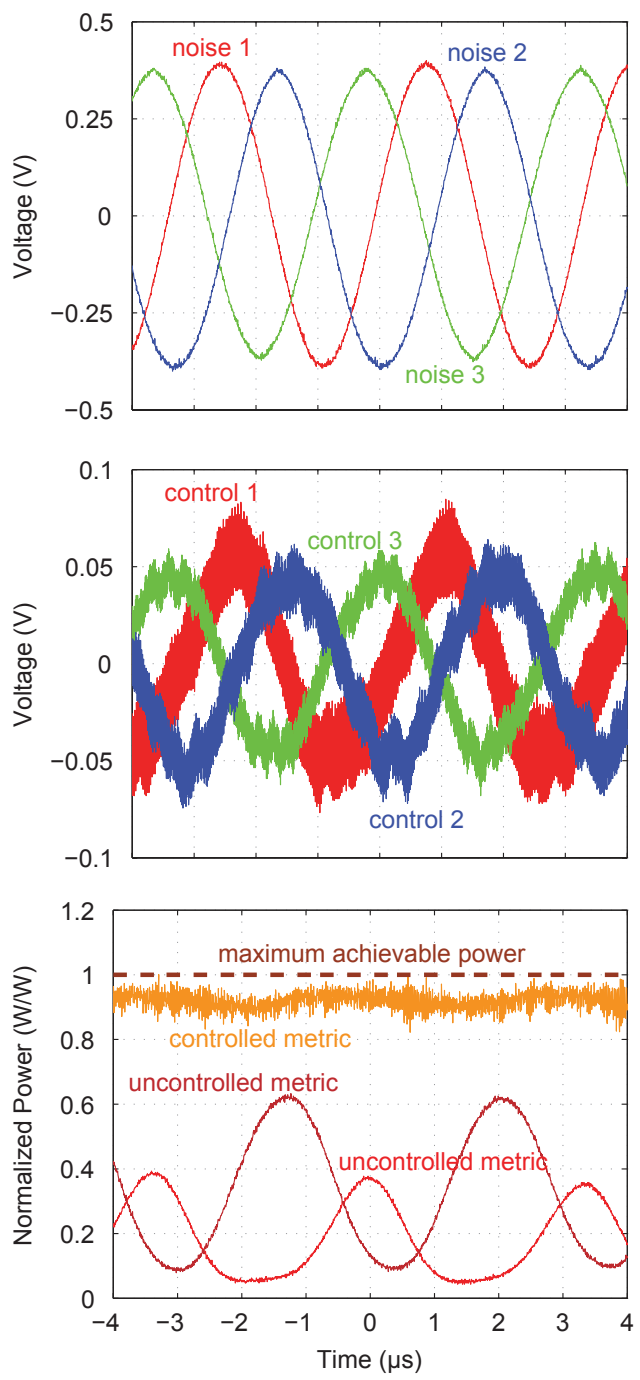

Fig. 8. Adaptation of 3 controlling outputs from the chip to externally applied sinusoidal phase noise in 3 different channels of the system. The sinusoidal phase distortions have frequencies of $290 \mathrm{kHz}, 295 \mathrm{kHz}$ and $300 \mathrm{kHz}$, and their peak-to-peak amplitude corresponds to a $\frac{\pi}{2}$ phase shift. The dither frequencies for the control outputs were set at $41 \mathrm{MHz}, 63 \mathrm{MHz}$ and $88 \mathrm{MHz}$.
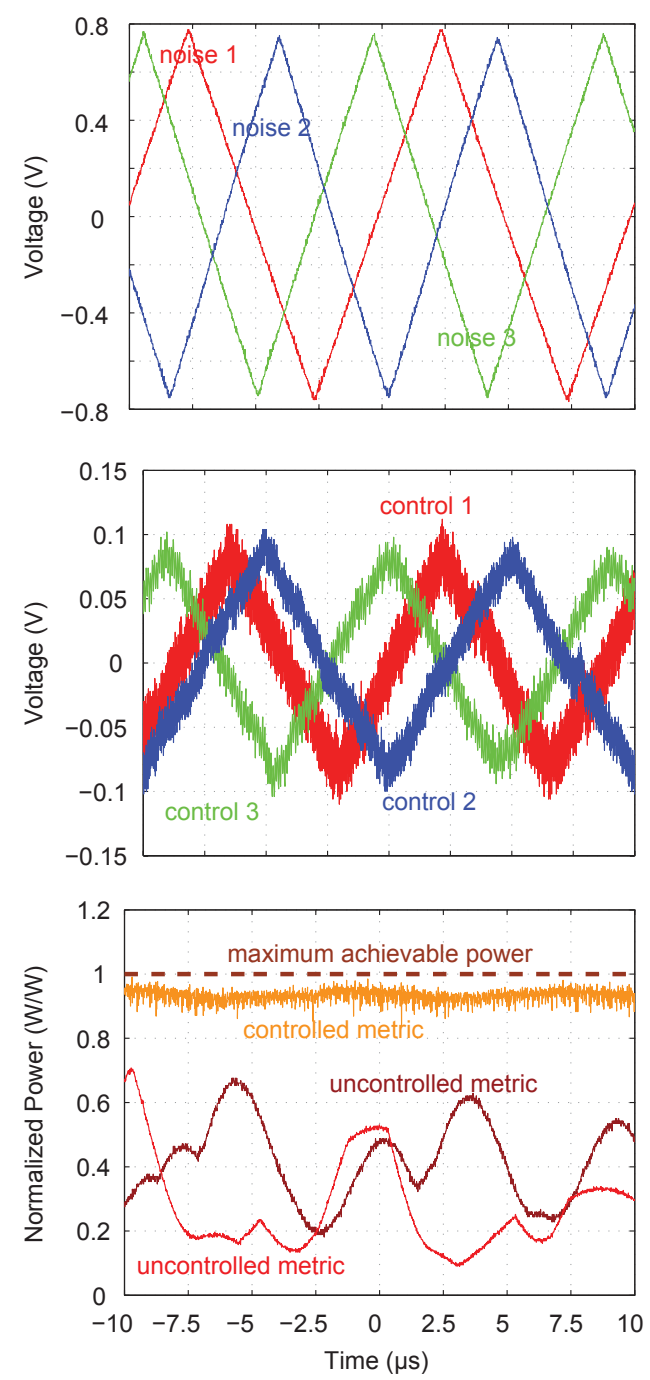

Fig. 9. Adaptation of 3 controlling outputs from the chip to externally applied triangular phase noise in 3 different channels of the system. The sinusoidal phase distortions have frequencies of $110 \mathrm{kHz}, 115 \mathrm{kHz}$ and $120 \mathrm{kHz}$, and their peak-to-peak amplitude corresponds to a $\pi$ phase shift. The dither frequencies for the control outputs were set at $41 \mathrm{MHz}, 63 \mathrm{MHz}$ and $88 \mathrm{MHz}$.

variable transformation $U_{i}=x_{i}+2 \kappa \pi, \kappa \in \mathbb{Z}$ and taking into account that $U_{i}=u_{i}+u_{i}^{\prime}$, we have that

$$
U_{i}=c+2 \kappa \pi \Leftrightarrow u_{i}=-u_{i}^{\prime}+c+2 \kappa \pi, \kappa \in \mathbb{Z} .
$$

From (6) it is readily deduced that in order to compensate for phase distortions, the phase shift introduced by the controller at each channel needs to cancel only the distortion at that channel. This phase shift can be a $2 \pi$ multiple of the distortion and will have a common mode component, $c$, pertinent to all channels. In the experimental setup used, no phase shift was introduced to one of the channels (Fig. 7), setting in this way $c=0$. Moreover, by enabling the " $2 \pi$ circuit", the possible values of $u_{i}$ were limited to within only two possible $2 \pi$ regions.

Fig. 8 shows the response of the controller when sinusoidal phase distortion of peak-to-peak amplitude equal to $\frac{\pi}{2}$ is introduced to 3 channels. The frequency of the distortion for each of the channels is $290 \mathrm{kHz}, 295 \mathrm{kHz}$ and $300 \mathrm{kHz}$. The dither frequencies of the controller were set at $41 \mathrm{MHz}, 63 \mathrm{MHz}$ and $88 \mathrm{MHz}$. The controlling voltages shown are the ac components of the output signals from the chip, prior to inverting amplification. These signals follow closely the introduced distortion and keep the received power at the photo-detector continuously within $90-95 \%$ of the maximum achievable value. Depicted are also two instances of the received power without any control.

Using the same configuration but this time with triangular phase distortion of $\pi$ peak-to-peak amplitude and frequency 
$110 \mathrm{kHz}, 115 \mathrm{kHz}$ and $120 \mathrm{kHz}$ for each of the 3 channels, the controller response was that of Fig. 9. The dither frequencies were again set at $41 \mathrm{MHz}, 63 \mathrm{MHz}$ and $88 \mathrm{MHz}$. Again, the controlling signals follow closely the applied distortion and bring the received power within $90-95 \%$ of the maximum achievable limit. Although not shown in the above figures, whenever drift in the phase modulators causes the applied phase distortion to exceed the thresholds of the " $2 \pi$ circuit", the control signals are instantaneously (in practice very fast) shifted by $2 \pi$ in a direction opposite to that of the drift.

The effect of the " $2 \pi$ circuit" in the control signals and the metric is shown in Fig. 10. For the experiment, distortion was applied to only one channel, while the common mode signal was set by a second channel. Control was introduced only to the phase-distorted channel with a dither frequency of $41 \mathrm{MHz}$. Phase-distortion was a sinusoidal signal of frequency $50 \mathrm{kHz}$ and peak-to-peak amplitude of $3 \pi$, triggering at every cycle the " $2 \pi$ circuit". In Fig. 10, the control signal has been appropriately scaled, so that the $2 \pi$ shifts are better pronounced with respect to the distortion. With the controller enabled, the received power stays within $80-90 \%$ of the maximum achievable limit. The effect of the $2 \pi$ shifts is minimal to the average power.

Experimental results from the use of the proposed integrated controller in a free-space optical setup have been demonstrated and reported in [22].

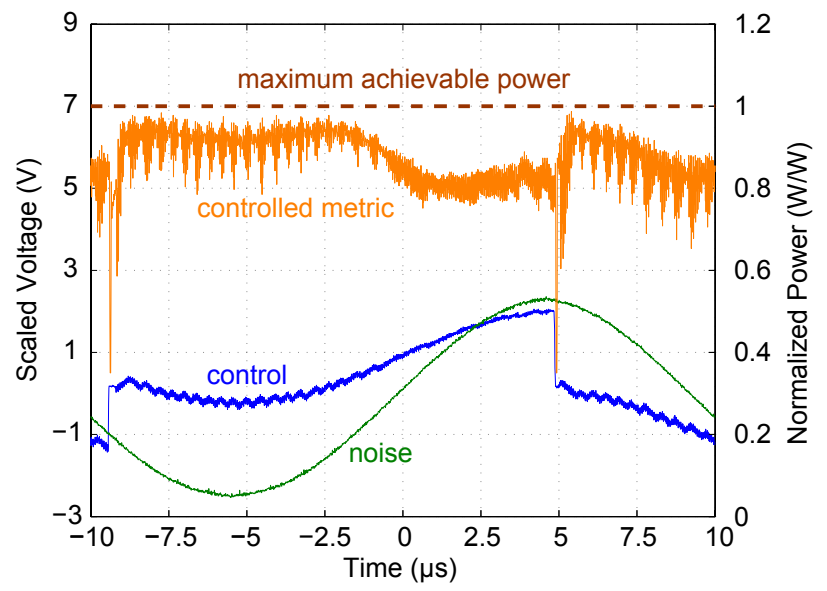

Fig. 10. Response of the system to an external sinusoidal phase noise of peak-to-peak amplitude equal to $3 \pi$ and frequency equal to $50 \mathrm{kHz}$, with the $2 \pi$ circuit enabled. At the points where the control is reset, the metric is instantaneously left uncontrolled. The dither frequency of the control was set to $41 \mathrm{MHz}$.

\section{CONCLUSION}

A mixed-signal architecture of an adaptive phase controller implementing the sinusoidal multi-dithering technique in VLSI has been presented. The architecture is meant for fast adaptive control in optical communications and is suitable for applications requiring high bandwidth phase noise cancellation, such as compensation of phase noise introduced by laser sources or optical amplifiers. The architecture performs adaptation in continuous-time by achieving compensation of the delay introduced by the loop. Moreover, it allows for phase control in multiple $2 \pi$ regions, a necessary feature for the case where performance metrics related in a modulo- $2 \pi$ fashion with the controlled phases are used. Experimental results in a fiber optic setup reveal compensation bandwidths in the range of several hundreds of $\mathrm{kHz}$, and minimal effect in the controlled performance metric during operation in multiple $2 \pi$ regions.

\section{ACKNOWLEDGMENTS}

The authors would like to thank Dr. Ernst Polnau for his help in conducting the experiments.

\section{REFERENCES}

[1] S. F. Clifford, G. M. B. Bouricius, G. R. Ochs, and M. H. Ackley, "Phase variations in atmospheric optical propagation," J. Opt. Soc. Am., vol. 61, no. 10, pp. 1279-1284, Oct. 1971.

[2] H. Matsumoto and K. Tsukahara, "Effects of the atmospheric phase fluctuation on long distance measurement," Applied Optics, vol. 23, no. 19, pp. 3388-3394, Oct. 1984.

[3] N. S. Nightingale and D. F. Buscher, "Interferometric seeing measurements at the La Palma observatory," Mon. Not. R. Astr. Soc., vol. 251, pp. 155-166, 1991.

[4] D. F. Buscher et al., "Interferometric seeing measurements on Mt. Wilson: power spectra and outer scales," Applied Optics, vol. 34, no. 6, pp. 1081-1096, Feb. 20, 1995

[5] K. Kikuchi, C.-E. Zah, and T.-P. Lee, "Measurement and analysis of phase noise generated from semiconductor optical amplifiers," IEEE J. Quantum Electron., vol. 27, no. 3, pp. 416-422, Mar. 1991.

[6] H. Bruesselbach et al., "Power-scalable phase-compensating fiber-array transceiver for laser communications through the atmosphere," J. Opt. Soc. Am. B, vol. 22, no. 2, pp. 347-353, Feb. 2005. 
[7] M. A. Vorontsov, G. W. Carhart, M. Cohen, and G. Cauwenberghs, "Adaptive optics based on analog parallel stochastic optimization: analysis and experimental demonstration," J. Opt. Soc. Am. A, vol. 17, no. 8, pp. 1440-1453, Aug. 2000.

[8] R. T. Edwards, M. Cohen, G. Cauwenberghs, M. A. Vorontsov, and G. W. Carhart, "Analog VLSI parallel stochastic optimization for adaptive optics," in Learning on Silicon, G. Cauwenberghs and M. A. Bayoumi, Eds. Boston, MA: Kluwer Academic, 1999, ch. 1, pp. 359-382.

[9] T. R. O'Meara, “The multidither principle in adaptive optics," J. Opt. Soc. Am., vol. 67, no. 3, pp. 306-315, Mar. 1977.

[10] W. B. Bridges et al., "Coherent optical adaptive techniques," Applied Optics, vol. 13, no. 2, pp. 291-300, Feb. 1974.

[11] G. A. Evans and J. M. Hammer, Surface emitting semiconductor lasers and arrays. Academic Press, Inc., 1993, ch. 8.

[12] A. Dembo and T. Kailath, "Model-free distributed learning," IEEE Trans. Neural Networks, vol. 1, no. 1, pp. 58-70, Mar. 1990

[13] D. N. Loizos, P. P. Sotiriadis, and G. Cauwenberghs, "A robust continuous-time multi-dithering technique for laser communications using adaptive optics," in Proc. Int. Symp. Circuits and Systems (ISCAS '06), May 2006, pp. 3626-3629.

[14] _ "BiCMOS multi-channel synchronous detection for sub- $\mu$ s adaptive control," IEEE J. Solid-State Circuits, submitted.

[15] K. Takizawa, H. Kikuchi, T. Aida, and M. Okada, "Optical parallel logic gate using a Pockels readout optical modulator and twisted nematic liquid-crystal cells," Opt. Lett., vol. 14, no. 4, pp. 208-210, Feb. 15, 1989.

[16] T. Hara, Y. Ool, Y. Suzuki, and M. H. Wu, "Transfer characteristics of the microchannel spatial light modulator," Applied Optics, vol. 28, no. 22, pp. 4781-4786, Nov. 15, 1989.

[17] K. Takizawa, "Analysis of electro-optic crystal-based Fabry-Perot etalons for high-speed spatial light modulators," Applied Optics, vol. 42, no. 6, pp. 1052-1067, Feb. 20, 2003.

[18] A. D. Fisher and C. Warde, "Technique for real-time high-resolution adaptive phase compensation," Opt. Lett., vol. 8, no. 7, pp. 353-355, July 1983.

[19] E. L. Wooten et al., "A review of lithium niobate modulators for fiber-optic communications systems," IEEE J. Select. Topics Quantum Electron., vol. 6, no. 1, pp. 69-82, Jan./Feb. 2000

[20] A. D. Fisher and C. Warde, "Simple closed-loop system for real-time optical phase measurement," Opt. Lett., vol. 4, no. 5, pp. 131-133, May 1979.

[21] T. H. Barnesa, T. Eijub, and K. Matsudab, "High resolution adaptive optics using an interference phase loop," Optics Commun., vol. 132, no. 5-6, pp. 494-502, Dec. 15, 1996.

[22] L. Liu, D. N. Loizos, P. P. Sotiriadis, and M. A. Vorontsov, "Coherent combining of multiple beams with multi-dithering technique: $100 \mathrm{kHz}$ closed-loop compensation demonstration," in Proc. of SPIE, vol. 6708, Aug. 26-30, 2007.

[23] L. Liu and M. A. Vorontsov, "Phase-locking of tiled fiber array using SPGD feedback controller," in Proc. of SPIE, vol. 5895, Sept. 1, 2005, pp. 58 950P-1-58 950P-9. 